\title{
Peripheral neuropathies during the COVID-19 pandemic: is there a relation?
}

\author{
Milena Trentinaglia ${ }^{1} \cdot$ Giuseppe Lippi ${ }^{2}$. Gian Luca Salvagno ${ }^{2,3} \cdot$ Marianna G. Rispoli $^{4}$ Maria Vittoria De Angelis ${ }^{4}$. \\ Francesca Castellani ${ }^{5}$. Daniela Alberti ${ }^{1}$. Giorgia T. Maniscalco ${ }^{6} \cdot$ Francesca Rossi $^{7} \cdot$ Mara Turri $^{8} \cdot$ Patrizia Rossi $^{9}$. \\ Elisabetta Del Zotto ${ }^{10}$. Simone Fusina ${ }^{11}$. Davide Cardellini ${ }^{12}$. Cecilia Zivelonghi ${ }^{1}$. Irene Volonghi ${ }^{13}$. \\ Salvatore Monaco ${ }^{1} \cdot$ Chiara Briani $^{5} \cdot$ Sergio Ferrari $^{1} \cdot$ Sara Mariotto $^{1}(\mathbb{0}$
}

Received: 13 December 2021 / Accepted: 24 February 2022 / Published online: 3 March 2022

(C) The Author(s), under exclusive licence to Springer Science+Business Media, LLC, part of Springer Nature 2022

\section{Introduction}

Neurological complications, including central and peripheral nervous system (PNS) disorders and myopathies, have been reported in up to $36.4 \%$ of hospitalised patients with coronavirus disease 2019 (COVID-19) [1]. Within PNS disorders, patients with neuralgic amyotrophy, critical illness polyneuropathy, positional compressive neuropathies, cranial neuropathies and especially Guillain-Barré syndrome (GBS) have been described [2]. The association between GBS and severe acute respiratory syndrome coronavirus-2 (SARS-CoV-2) infection was not unexpected, due to the post-infectious nature of the disease, possibly sustained by immune-mediated antibody-response induced by molecular mimicry [3].

However, discordant data have been reported on the incidence of GBS during the COVID-19 pandemic and, therefore, on a possible correlation between these two conditions $[4,5]$. In addition, the association between SARS-CoV-2

Sara Mariotto

sara.mariotto@gmail.com

1 Neurology Unit, Department of Neurosciences, Biomedicine, and Movement Sciences, University of Verona, Policlinico GB Rossi, P.le LA Scuro 10, 37134 Verona, Italy

2 Section of Clinical Biochemistry, University of Verona, Verona, Italy

3 Service of Laboratory Medicine, Pederzoli Hospital, Peschiera del Garda, Verona, Italy

4 Department of Neurology, SS Annunziata Hospital, Chieti, Italy

5 Department of Neurosciences, University of Padova, Padova, Italy

6 Department of Neurology and Stroke Unit, "A. Cardarelli Hospital", Naples, Italy infection and other immune-mediated neuropathies has not been addressed so far. This study is aimed to report the clini$\mathrm{cal} /$ paraclinical characteristics of patients with new onset/ relapse of PNS disorders of suspected autoimmune origin occurred during the COVID-19 pandemic and to determine their relation with antecedent SARS-CoV-2 infection through extensive serological analysis.

\section{Methods}

\section{Study subjects}

We retrospectively identified consecutive inpatients and outpatients with acute onset/relapse of PNS disorders of suspected autoimmune origin referred to the Laboratory of Neuropathology, University Hospital of Verona, Italy, for serological testing aimed at detecting autoantibodies against myelin associated glycoprotein (MAG $n=19$ ), intracellular/

7 Neurology Unit, Mater Salutis Hospital, Legnago, Verona, Italy

8 Department of Neurology/Stroke Unit, San Maurizio Hospital, Bolzano, Italy

9 Neurology Unit, St Bassano Hospital, Bassano del Grappa, Vicenza, Italy

10 Neurology Unit, Poliambulanza Hospital, Brescia, Italy

11 Neurology Unit, S. Bonifacio Hospital, Verona, Italy

12 Neurology Unit, Ca' Foncello Hospital, Treviso, Italy

13 Neurology Unit, Department of Clinical and Experimental Sciences, University of Brescia, Brescia, Italy 
synaptic antigens $(n=15)$ and/or gangliosides $(n=41)$, between January 1st and December 31st, 2020.

\section{SARS-CoV-2 IgA/lgG testing}

All sera were tested using an FDA-approved ELISA assay for detecting anti-SARS-CoV-2 (S1 subunit of the spike protein) IgA and IgG (Euroimmun, Luebeck, Germany). Positive results were validated using a trimeric anti-SARSCoV-2 S1/S2 IgG test (DiaSorin, Saluggia, Italy), an antiSARS-CoV-2 Receptor Binding Domain (RBD) IgG test (Beckman-Coulter, Brea, USA) and an anti-SARS-CoV-2 RBD total antibodies test (Roche Diagnostics, Basel, Switzerland). Available CSF samples from SARS-CoV-2 seropositive cases $(n=2)$ were also tested, as previously described (at 1:10 dilution) [6].

\section{Antibody testing}

IgM antibodies to MAG were detected with a quantitative ELISA assay (Bühlmann Laboratories AG, Schönenbuch, Switzerland), whilst gangliosides (GM1 IgM/IgG, GM2 $\mathrm{IgM} / \mathrm{IgG}$, GD1a IgM/IgG, GD1b IgM/IgG, GQ1b IgG and sulfatide $\operatorname{IgM}$ ) were analysed using a home-made ELISA assay [7]. Serum autoantibodies against intracellular/synaptic antigens including $\mathrm{Hu}, \mathrm{Ri}$, Yo, Amphiphysin, PNMA2, CV2, Recoverin, SOX1, Titin, Zic4, GAD65 and Tr were assessed with line blot (Euroimmun, Luebeck, Germany) and confirmed with a tissue-based assay for intracellular antigens on frozen sections of rat brain [8].

\section{Clinical and paraclinical data}

We collected demographic, clinical, neurophysiological and laboratory features, as well as treatment and outcome (graded according to ONLS score) data of all included cases with a standardised case report form. GBS diagnosis was stated using previously established diagnostic criteria [9] whilst the diagnosis of anti-MAG-related neuropathy, chronic inflammatory demyelinating polyneuropathy (CIDP) and multifocal motor neuropathy was defined according to the revised European Federation of Neurological Societies/ Peripheral Nerve Society Guidelines.

\section{Statistical analysis}

For comparison between SARS-CoV-2 IgA/IgG seropositive and seronegative subjects, differences in nominal categorical variables were assessed with Fisher's exact test and in ordinal ones with $Z$-test, whilst Student's $T$-test was used for continuous variables, applying Welch's correction for unequal variance, when appropriated. Mann-Whitney test was applied to compare median values. For all analyses, a $p$ value $<0.05$ was considered significant.

\section{Results}

A total number of 47 patients referred from 8 different Italian hospitals were included in the study based on the aforementioned inclusion criteria. The median age was 56 (range $14-81$ ) years with male predominance (29/47 cases; $62 \%)$. Most of included cases had acute onset during the inclusion timeframe, and only 2 patients presented a relapse of a previously diagnosed CIDP. Neurological diagnoses in these patients were as follows: GBS $(n=15)$, CIDP $(n=7)$, MAGassociated neuropathy $(n=3)$, multifocal motor neuropathy $(n=1)$, neuropathy associated with systemic disorders (cancer $n=4$, rheumatoid arthritis $n=2$ ) and idiopathic neuropathy $(n=15)$. Only patients with PNS disorders of suspected autoimmune origin were included and none required admission to intensive care unit during the acute stage, so that none of included cases had factors suggestive of critical illness polyneuropathy or positional compressive neuropathy.

We identified SARS-CoV-2 IgA and IgG in 7 out of 47 (14.9\%) patients, with no discrepancy on confirmatory analyses. No paired CSF positivity was detected. A known antecedent SARS-CoV-2 infection determined with a positive SARS-CoV-2 PCR on nasopharyngeal swab was reported in 5 out of 7 patients, 2 of whom needed hospitalization. All patients with known SARS-CoV-2 infection developed neurological symptoms after COVID-19, with median time lapse between neurological symptoms onset and SARSCoV-2 systemic symptoms of 34 (range 12-70) days. The remaining 2 patients never reported symptoms/signs of SARS-CoV-2 infection.

Demographic, clinical, neurophysiological and laboratory data of the 7 SARS-CoV-2 seropositive cases are reported in Table 1.

Mean age of seropositive patients was 60 (range 48-78) years, and 3 were females. Neurological diagnoses were GBS (acute motor axonal neuropathy, AMAN $n=1$, acute inflammatory demyelinating polyradiculoneuropathy, AIDP $n=1)$, CIDP $(n=2)$ and idiopathic neuropathies $(n=3), 2$ of whom had previous symptomatic COVID-19. Electroneurography (ENG) was abnormal in 6/7 patients (demyelinating $n=2$, axonal $n=2$, demyelinating and axonal $n=2$ ). CSF analysis was performed in all GBS/CIDP patients, showing pleocytosis in one and elevated protein content in all cases. Median ONLS score was $3.29(0-10)$ at onset, and $3.14(0-11)$ after hospital discharge, respectively. Neural autoantibody testing was negative in all but 1 patient with CIDP, who tested positive for anti-GD1b and anti-GM2 IgM antibodies. Among SARS-CoV-2 seronegative cases, we detected anti-GM1 IgM and/or IgG $(n=3)$, GM2 IgM 


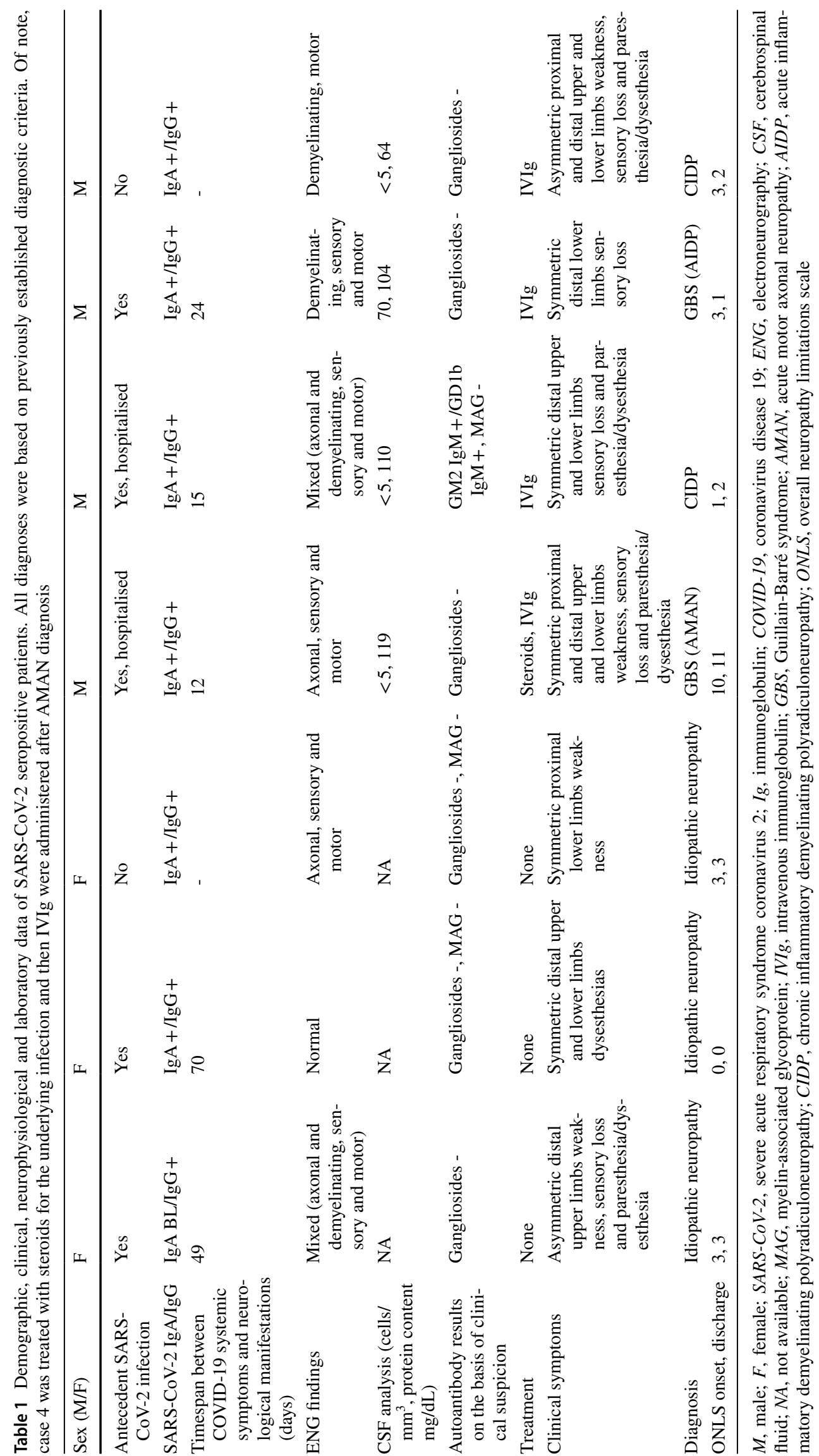


$(n=1)$, sulfatides $\operatorname{IgM}(n=1)$, anti-MAG $(n=3)$ and antirecoverin $(n=1)$.

No significant differences emerged by comparing clinical and paraclinical features between SARS-CoV-2 seropositive vs seronegative cases (Table 2). Notably, antibodies to MAG and intracellular/synaptic antigens could only be detected in a subgroup of SARS-CoV-2 seronegative cases.

\section{Discussion}

By analysing the association between SARS-CoV-2 infection and immune-mediated neuropathies, we herein observed that (i) a significant number of patients with PNS disorders of suspected autoimmune origin occurring during the COVID-19 pandemic had antecedent SARS-CoV-2 infection; (ii) peripheral neuropathies are not always associated with severe COVID-19 but may also occur in cases without systemic symptoms; (iii) idiopathic peripheral neuropathy is the most common subtype of PNS disorders occurring after SARS-CoV-2 infection (GBS can also occur and is
Table 2 Comparison of clinical and paraclinical features between SARS-CoV-2 seropositive vs seronegative patients

\begin{tabular}{|c|c|c|c|}
\hline & $\begin{array}{l}\text { SARS-CoV-2 } \\
\text { seropositive patients } \\
(n=7)\end{array}$ & $\begin{array}{l}\text { SARS-CoV-2 } \\
\text { seronegative patients } \\
(n=40)\end{array}$ & $p$-value \\
\hline \multicolumn{4}{|l|}{ Demographic data } \\
\hline Age, median (range) & $60.1(48-78)$ & $55.8(14-81)$ & 0.496 \\
\hline Female, $n(\%)$ & $3(43 \%)$ & $15(38 \%)$ & 1 \\
\hline \multicolumn{4}{|l|}{ Clinical history, $n(\%)$} \\
\hline Haematological diseases & 0 & $8(20 \%)$ & 0.329 \\
\hline Tumours & 0 & $4(10 \%)$ & 1 \\
\hline \multicolumn{4}{|l|}{ Final diagnosis, $n(\%)$} \\
\hline GBS & $2(28.6 \%)$ & $13(31 \%)$ & 1 \\
\hline CIDP & $2(28.6 \%)$ & $5(13 \%)$ & 0.276 \\
\hline Anti-MAG-related neuropathy & 0 & $3(8 \%)$ & 1 \\
\hline Multifocal motor neuropathy & 0 & $1(2.5 \%)$ & 1 \\
\hline Neuropathy correlated with systemic conditions & 0 & $6(15.5 \%)$ & 0.571 \\
\hline Idiopathic neuropathy & $3(42.8 \%)$ & $12(30 \%)$ & 0.664 \\
\hline \multicolumn{4}{|l|}{ ENG findings, $n /$ total $(\%)$} \\
\hline Demyelinating & $2 / 7(29 \%)$ & $19 / 37(51 \%)$ & 0.416 \\
\hline Axonal & $2 / 7(29 \%)$ & $10 / 37(27 \%)$ & 1 \\
\hline Demyelinating and axonal & $2 / 7(29 \%)$ & $7 / 37(19 \%)$ & 0.619 \\
\hline Normal* & $1 / 7(14 \%)$ & $1 / 37(3 \%)$ & 0.296 \\
\hline Sensory & $0 / 7$ & $2 / 37(5 \%)$ & \\
\hline Motor & $1 / 7(14 \%)$ & $11 / 37(30 \%)$ & 0.653 \\
\hline Sensory and motor & $5 / 7(71 \%)$ & $23 / 37(62 \%)$ & 1 \\
\hline \multicolumn{4}{|l|}{ CSF analysis, $n /$ total (\%) } \\
\hline Pleocytosis & $1 / 4(25 \%)$ & $11 / 30(37 \%)$ & 1 \\
\hline Increased protein content & $4 / 4(100 \%)$ & $20 / 30(67 \%)$ & 0.296 \\
\hline Protein content (mg/dL), median (range) & $99.25(64-119)$ & $98.15(20-284)$ & 0.952 \\
\hline \multicolumn{4}{|l|}{ Autoantibody testing, $n /$ total (\%) } \\
\hline Anti-gangliosides & $1 / 7(14 \%)$ & $5 / 34(15 \%)$ & 1 \\
\hline Anti-MAG & $0 / 3$ & $3 / 16(18.7 \%)$ & 1 \\
\hline Anti-onconeural & $0 / 0$ & $1 / 15(7 \%)$ & - \\
\hline
\end{tabular}

$n$, number; \%, percentage; $S A R S-C o V-2$, severe acute respiratory syndrome coronavirus 2; GBS, GuillainBarré syndrome; $C I D P$, chronic inflammatory demyelinating polyradiculoneuropathy; $M A G$, myelin-associated glycoprotein; $E N G$, electroneurography; $C S F$, cerebrospinal fluid

*The SARS-CoV-2 seropositive patient had a clinical phenotype suggestive for small fibre neuropathy whilst ENG was conducted at the early stage of the disease in the SARS-CoV-2 seronegative patient later diagnosed with GBS (AIDP) 
as common as CIDP); (iv) PNS disorders occurring after SARS-CoV-2 infection do not present a distinct phenotype; and (v) autoantibodies to nervous system antigens are rarely observed in patients with PNS disorders and previous SARS-CoV-2 infection.

In particular, we noted a higher incidence of SARSCoV-2 IgG seropositivity in our cohort (14.9\%) than that reported by epidemiological data of general and local population in the analysed timeframe $(2.5-2.6 \%)[10,11]$. Although referral bias and regional differences must be considered, this discrepancy suggests a possible association between SARS-CoV-2 and inflammatory PNS conditions.

In addition, previous studies mainly focused on describing PNS disorders occurring in patients with well-known SARS-CoV-2 infection [12]. The unique design of our study allowed the identification of peripheral neuropathies in patients with antecedent asymptomatic SARS-CoV-2 infection, expanding the few cases previously described with mild systemic symptoms [13] and supporting clinicians to consider this viral disease as a potential trigger of PNS disorders also in patients without a known history of COVID-19.

Then, in agreement with earlier studies, GBS was recognised as one of the PNS conditions potentially triggered by SARS-CoV-2 infection [2, 12-14]. However, according to our data, CIDP is as common as GBS among patients with an antecedent SARS-CoV-2 infection, which might support clinicians to consider this infectious disease as a potential trigger also in patients with a subacute onset and chronic course. In addition, 3/7 cases could not be defined according to established diagnostic criteria and were classified as "idiopathic peripheral neuropathy", thus suggesting that the spectrum of PNS disorders possibly related to SARS-CoV-2 infection might be wider than expected, particularly when considering systemic asymptomatic cases, which could have been underestimated at present.

Notably, in our cohort, median latency between neurological symptoms and COVID-19 in cases with a known SARS-CoV-2 infection was longer (34 days) than that previously reported (3-33 days) [2, 14], supporting the occurrence of a post-infectious immune-response rather than a para-infectious aetiology.

Finally, in agreement with previous studies, we confirmed that anti-gangliosides antibodies are often negative in patients with peripheral neuropathies occurring after SARSCoV-2 infection $[14,15]$ and we expanded these findings providing evidence of absence of other well-known antibodies. According to this observation, cross-reactivity between COVID-19 epitopes and peripheral nerve antigens does not explain the correlation between the two syndromes.

To conclude, SARS-CoV-2 infection should be included among the possible trigger of PNS disorders, also in asymptomatic cases. These patients have a heterogenous phenotype and are usually negative for well-known autoantibodies to
PNS antigens. Our study is limited by the low number of included patients and prospective multicentre studies with prolonged follow-up over different timespan of COVID-19 pandemic are needed to confirm and expand our findings.

Author contribution MT: clinical data collection, data generation and interpretation, drafting the manuscript; GL: analysis of samples, results interpretation, revising the manuscript for intellectual content; GLS: analysis of samples and results interpretation; MGR, MVDA, FC, GTM, FR, MT, PR, EDZ, SF, DC, IV: sample and clinical data collection and interpretation; DA: analysis of samples and data interpretation; CZ: data analysis and interpretation; SMo data generation and interpretation, revising the manuscript for intellectual content; CB: sample and clinical data collection, data generation and interpretation, revising the manuscript for intellectual content; SF: sample and clinical data collection, design and conceptualization of the study, revising the manuscript for intellectual content; SMa: design and conceptualization of the study, sample and clinical data collection, data generation and interpretation, drafting the manuscript. All authors read and approved the final manuscript.

Availability of data and material The data that support this study are available for sharing and further examinations from the corresponding author (S.M.) on reasonable requests. The data are not publicly available because they contain information that could compromise patients' consent.

Code availability Not applicable.

\section{Declarations}

Conflict of interest S. Ferrari received support for attending scientific meetings by Shire, Sanofi Genzyme and Euroimmun. S. Mariotto received support for attending scientific meetings by Merck and Euroimmun and received speaker honoraria from Biogen. The other authors report no competing interests.

Ethics approval, consent to participate and consent for publication The study was approved by the local Bioethics Committee (Comitato Etico per la Sperimentazione Clinica, Azienda Ospedaliera Universitaria Integrata di Verona, BIOB-NEU-DNA-2014, protocol 13582).

\section{References}

1. Mao L, Jin H, Wang M, et al. Neurologic manifestations of hospitalized patients with coronavirus disease 2019 in Wuhan. China JAMA Neurol. 2020;77:683-90. https://doi.org/10.1001/jaman eurol.2020.1127.

2. Finsterer J, Scorza FA, Fiorini AC. SARS-CoV-2-associated Guillain-Barre syndrome in 62 patients. Eur J Neurol. 2021;28:e10-2. https://doi.org/10.1111/ene.14544.

3. Jacobs BC, Rothbarth PH, van der Meché FG, et al. The spectrum of antecedent infections in Guillain-Barré syndrome: a case-control study. Neurology. 1998;51:1110-5. https://doi.org/10.1212/ wnl.51.4.1110.

4. Keddie S, Pakpoor J, Mousele C, et al. Epidemiological and cohort study finds no association between COVID-19 and Guillain-Barré syndrome. Brain. 2021;144:682-93. https://doi.org/10.1093/brain/ awaa433. 
5. Fragiel M, Miró Ò, Llorens P, et al. Incidence, clinical, risk factors and outcomes of Guillain-Barré in Covid-19. Ann Neurol. 2021;89:598-603. https://doi.org/10.1002/ana.25987.

6. Alexopoulos H, Magira E, Bitzogli K, et al. Anti-SARS-CoV-2 antibodies in the CSF, blood-brain barrier dysfunction, and neurological outcome: studies in 8 stuporous and comatose patients. Neurol Neuroimmunol Neuroinflamm. 2020;7: e893. https://doi. org/10.1212/NXI.0000000000000893.

7. Franciotta D, Gastaldi M, Benedetti L, et al. Diagnostics of dysimmune peripheral neuropathies. Neurol Sci. 2017;38:243-7. https:// doi.org/10.1007/s10072-017-3025-3.

8. Ricken G, Schwaiger C, De Simoni D, et al. Detection methods for autoantibodies in suspected autoimmune encephalitis. Front Neurol. 2018;9:841. https://doi.org/10.3389/fneur.2018.00841.

9. Willison HJ, Jacobs BC, van Doorn PA. Guillain-Barré syndrome. Lancet. 2016;388:717-27. https://doi.org/10.1016/S01406736(16)00339-1.

10. Guerriero M, Bisoffi Z, Poli A, et al. Prevalence of SARS-CoV-2, Verona, Italy, April-May 2020. Emerg Infect Dis. 2021;27:229_ 32. https://doi.org/10.3201/eid2701.202740.

11. ISTAT, Ministero della Salute. Primi risultati dell'indagine di sieroprevalenza sul SARS-CoV-2. $2020: 10$. https://www.istat. it/it/files//2020/08/ReportPrimiRisultatiIndagineSiero.pdf [access date: July 2021]
12. Guerrero JI, Barragán LA, Martínez JD, et al. Central and peripheral nervous system involvement by COVID-19: a systematic review of the pathophysiology, clinical manifestations, neuropathology, neuroimaging, electrophysiology, and cerebrospinal fluid findings. BMC Infect Dis. 2021;21:515. https://doi.org/10.1186/ s12879-021-06185-6.

13. Scheidl E, Canseco DD, Hadji-Naumov A, et al. Guillain-Barré syndrome during SARS-CoV-2 pandemic: a case report and review of recent literature. J Peripher Nerv Syst. 2020;25:204-7. https://doi.org/10.1111/jns.12382.

14. Uncini A, Vallat JM, Jacobs BC. Guillain-Barré syndrome in SARS-CoV-2 infection: an instant systematic review of the first six months of pandemic. J Neurol Neurosurg Psychiatry. 2020;91:1105-10. https://doi.org/10.1136/jnnp-2020-324491.

15. Toscano G, Palmerini F, Ravaglia S, et al. Guillain-Barré syndrome associated with SARS-CoV-2. N Engl J Med. 2020;382:2574-6. https://doi.org/10.1056/NEJMc2009191.

Publisher's note Springer Nature remains neutral with regard to jurisdictional claims in published maps and institutional affiliations. 